\title{
Нестандартные лапароскопические операции при технически сложных
} холецистәктомиях

\author{
V. V. HRUBNYK, A. I. TKACHENKO, M. V. PRYKUPENKO, K. O. VOROTYNTSEVA \\ Odesa National Medical University
}

\section{NON-STANDARD LAPAROSCOPIC SURGERIES AT TECHNICALLY DIFFICULT CHOLECYSTECTOMY}

\begin{abstract}
В данной работе мы сравнили результаты использования лапароскопических холецистэктомий при лечении 5648 больных. По поводу острого холецистита прооперировано 48 \% пациентов. В период с 2005 по 2008 год использовали стандартную тактику лечения и переходили к открытым операциям при возникновении технических сложностей во время проведения лапароскопических холецистэктомий. В период с 2009 по 2012 год применяли нестандартную технику лечения, в технически сложных случаях выполняли лапароскопическую субтотальную холецистэктомию. Данная операция позволит избежать целого ряда серьёзных интраоперационных повреждений жёлчных протоков, позволит снизить частоту раневых инфекций и смертность, уменьшится длительность пребывания пациентов в стационаре.
\end{abstract}

In this work we compared results of laparoscopic cholecystectomy application at treatment of 5648 patients. 48 \% patients were operated because of an acute cholecystitis. From 2005 to 2008 it was used the standard treatment tactics and were developed open surgeries when rising the technical complication during performance of laparoscopic cholecystectomy. From 2009 to 2012 it was used the non-standard treatment technique, in technically complicated cases it was used the laparoscopic subtotal cholecystectomy. This surgery allows to avoid the whole raw of serious intraoperative lesions of bile ducts, to reduce the frequency of wound infections and morbidity, to decrease the duration of patients' stay in the hospital.

Постановка проблемы и анализ последних исследований и публикаций. В настоящее время в большинстве стран лапароскопическая холецистэктомия стала "золотым стандартом" в желчнокаменной болезни (ЖКБ) [1, 4]. “Ахиллесовой пятой” этой методики является достаточно высокая частота повреждения желчных протоков, которая в 3-5 раз превышает показатели при открытых операциях. По данным мультицентрических исследований, проведенных ассоциациями лапароскопических хирургов во многих развитых странах Европы, частота повреждения желчных протоков составляет 0,6-1,2\% [3, 7, 9, 12]. Для безопасного выполнения лапароскопической холецистэктомии необходимо провести аккуратную диссекцию в области треугольника Кало с выделением и четкой идентификацией пузырного протока и артерии. При острых деструктивных формах ЖКБ, при хроническом воспалительном рубцовом процессе в области гепатодуоденальной связки, при наличии синдрома Мириззи, при определенных особенностях анатомического строения пече- ночного дерева этот этап операции произвести достаточно четко и безопасно не удается [6]. Поэтому возникает опасность повреждения внепеченочных желчных протоков и крупных сосудов. Стандартным выходом при таких ситуациях большинство хирургов предлагает выполнение конверсии, которая составляет от 30 до $50 \%$ $[7,10,14,21]$. Однако даже в случаях, когда выполняется конверсия, не всегда возможно идентифицировать пузырный проток и артерию, поэтому даже при конверсии остается опасность повреждения желчных протоков. Нестандартным выходом из положения при выполнении технически сложных лапароскопических холецистэктомий является проведение субтотального удаления желчного пузыря с иссечением всей его передней стенки и кармана Гартмана [7]. К сожалению, далеко не все хирурги знают и владеют этой методикой [11].

Цель работы: изучение эффективности использования лапароскопической субтотальной холецистэктомии при выполнении технически сложных холецистэктомий. 
Материалы и методы. При проведении данного исследования мы сравнили результаты использования лапароскопических холецистэктомий при лечении 5648 больных. 48 \% пациентов оперировались нами по поводу острого холецистита. Среди оперируемых больных преобладали женщины, их было 68 \%. Возраст больных варьировал в широких пределах - от 13 до 92 лет. Средний возраст больных составил $(58,6 \pm 12,5)$ года. В первый период с 2005 по 2008 год мы использовали стандартную тактику лечения и переходили к открытым операциям при возникновении технических сложностей во время проведения лапароскопических холецистэктомий. Во второй период с 2009 по 2012 год мы применяли нестандартную тактику лечения, в технически сложных случаях выполняли лапароскопическую субтотальную холецистэктомию.

Для выполнения лапароскопической операции использовалась стандартная техника с введением 4 троакаров. Изначально выполнялась попытка диссекции треугольника Кало. В случаях, когда достоверно идентифицировать пузырный проток и пузырную артерию не удавалось, принималось решение приступить к выполнению субтотальной лапароскопической холецистэктомии. После пункции и эвакуации содержимого желчного пузыря, с помощью диатермического крючка или ультразвуковых ножниц рассекалась стенка пузыря, зажимом извлекались все конкременты, затем, используя ультразвуковые инструменты, выполнялось иссечение свободной части желчного пузыря. Иссечь стенку желчного пузыря из ложа не пытались ни в одном случае, так как выполнение данного этапа операции могло привести к серьезной травме желчных протоков и выраженному кровотечению. Операцию заканчивали дренированием подпеченочного пространства.

Результаты исследований и их обсуждение. За первый период нами прооперировано 2720 пациентов с выполнением лапароскопических холецистэктомий. Технически сложные случаи выявлены у 42 больных (1,5\%). Данным пациентам была произведена конверсия с последующей холецистэктомией. Повреждение желчных протоков за этот период было у 3 больных $(0,1 \%)$. Такие осложнения, как желчный перитонит, поддиафрагмальный и подпеченочный абсцессы, наблюдались у 25 больных $(0,9 \%)$. Умерли по различным причинам 4 пациенты $(0,1 \%)$. Во второй период нами прооперировано 2928 пациентов. Технически сложные ситуации возникли у 45 пациентов $(1,5 \%)$. Конверсия произведена всего у 4 пациентов $(0,1 \%)$. Причинами конверсии служили желчно-дуоденальный свищ у 2 пациентов, синдром Мириззи у 2 пацентов. У 41 больного (1,5 \%) была выполнена лапароскопическая субтотальная холецистэктомия. Среди данных больных было 28 женщин и 13 мужчин. Средний возраст пациентов составил $(68 \pm 7,6)$ года. Среднее время операции составило 95 мин (от 45 до 130 мин). Показаниями для перехода к субтотальной холецистэктомии был тяжелый фиброз в области треугольника Кало с невозможностью идентифицировать анатомические структуры (21 пациент), выраженный воспалительный процесс желчного пузыря (10 пациентов), эмпиема желчного пузыря (5 пациентов), перфорация желчного пузыря (4 пациента), гангрена желчного пузыря (5 пациентов). В среднем интраоперационная кровопотеря составила 80 мл (от 20 до 230 мл), что объясняется широким использованием ультразвуковых ножниц с хорошей коагуляционной способностью. Просвет желчного пузыря у 17 пациентов коагулировался изнутри кармана Гартмана с помощью ультразвуковых ножниц. У 8 пациентов произведено прошивание кармана Гартмана в области перехода в пузырный проток. У остальных пациентов просвет желчного пузыря не прошивали, а подводили дренажную трубку, которая фиксировалась к тканям кармана Гартмана. Длительное истечение желчи (более 5 дней) наблюдалось у 5 пациентов, данным больным была выполнена эндоскопическая ретроградная холангиопанкреатография (ЭРХПГ) с эндоскопической папиллотомией (ЭП) и удалением конкрементов из холедоха, после чего желчеистечение прекратилось. Желчного перитонита не было ни в одном случае. У двух больных наблюдалось формирование подпеченочного и поддиафрагмального абсцессов, которые были успешно дренированы под контролем УЗИ. Всего в раннем послеоперационном периоде осложнения наблюдались у 6 больных (14,6 \%): желчеистечение было у 3 пациентов, формирование абсцессов - у 2, послеоперационная пневмония и плеврит - у одного пациента.

Послеоперационный период протекал по-разному в зависимости от характера основной патологии. Длительность нахождения в стационаре пациентов с острым холециститом составила в среднем 8 дней, с выполнением трёх ЭРХПГ. При хроническом холецистите пациенты находились в стационаре в среднем 6 дней. Длительность дренирования брюшной полости в среднем составила 5 дней (от 2 до 13 дней).

Обсуждение. Выполнение лапароскопической субтотальной холецистэктомии является альтернативой конверсии, в случаях когда лапароскопически невозможно идентифицировать пузырный про- 
ток и пузырную артерию. При выполнении данной операции отсутствует повреждение желчных протоков. Почти три четверти пациентов данного исследования были выписаны из стационара в течение одной недели после операции.

Ряд исследований определил показания для перехода от обычной лапароскопической холецистэктомии к субтотальной. Основными причинами выполнения субтотальной холецистэктомии являются воспалительный процесс и фиброз треугольника Кало, когда невозможно четко определиться в анатомических структурах и риск повреждения желчных протоков очень велик [13, 18-20]. Данные этих исследований показывают что при выполнении этой операции средний период нахождения пациентов в стационаре составляет от 3 до 10 дней, в то время как данные нашего исследования показывают, что средняя продолжительность пребывания пациентов в стационаре составила 5 дней.

Частота осложнений при выполнении субтотальной холецистэктомии составляет 16,6 \%, в то время как при выполнении конверсии частота развития раневых инфекций увеличивается на $14 \%$, желчеистечения - на 14 \% и осложнения со стороны сердечно-сосудистой системы - на $9 \%$ [1, 15, 18]. Кроме того, увеличивается время нахождения пациентов в стационаре [13, 18-20]. Важным преимуществом лапароскопической субтотальной холецистэктомии является возможность выполнения данной операции у пациентов с нарушениями со стороны сердечно-сосудистой системы [8].

Ни у одного пациента данного исследования не было раневых осложнений, этот факт можно

\section{СПИСОК ЛИТЕРАТУРЫ}

1. Predictive factors for conversion of laparoscopic cholecystectomy / A. Alponat, C. K. Kum, B. C. Koh [et al.] // World Journal of Surgery. - 1997. - Vol. 21. - P. 629-633.

2. Beldi G. Laparoscopic cholecystectomy for severe cholecystitis: a follow-up study / G. Beldi, A. Glattli // Surgical Endoscopy. 2003. - Vol. 17. - P. 1437-1439.

3. Berggren U. Laparoscopic versus open cholecystectomy: hospitalisation, sick leave, and trauma responses / U. Berggren, T. Gordh, D. Grama [et al.] // British Journal of Surgery. - 1994. Vol. 81. - P. 1362-1365.

4. Bickel A. Laparoscopic subtotal cholecystectomy / A. Bickel, B. Shtamler // Journal of Laparoendoscopic Surgery. - 1993. Vol. 3. - P. 365-367.

5. Laparoscopic subtotal cholecystectomy: a review of 56 procedures / P. K. Chowbey, A. Sharma, R. Khullar [et al.] // Journal of Laparoendoscopic \& Advanced Surgical Techniques. 2000. - Vol. A10. - P. 31-34.

6. Chuang S. C. Risk factors for wound infection after cholecystectomy / S. C. Chuang, K. T. Lee, W. T. Chang [et al] // Journal of the Formosan Medical Association. - 2004. - Vol. 103. P. 607-612. объяснить относительно небольшим количеством пациентов, однако данные литературы показывают, что при выполнении открытых операций частота раневых осложнений увеличивается на порядок [6].

Ряд хирургов при выполнении лапароскопической субтотальной холецистэктомии, кроме иссечения свободной стенки желчного пузыря, производит закрытие пузырного протока, используя швы, клипсы, скрепки [4, 5, 9, 15, 17]. Результаты исследований, при которых выполнялось коагулирование пузырного протока, показывают, что частота желчеистечения была снижена до 3-5 \% [5, 9].

Лапароскопическая субтотальная холецистэктомия не является рутинной операцией, и для ее выполнения необходимо наличие высококвалифицированных опытных хирургов, однако опыт применения данной операции показал, что использование данной методики позволит избежать повреждения желчных протоков в самых сложных чрезвычайных ситуациях.

Выводы. Лапароскопическая субтотальная холецистэктомия является нестандартной тактикой в случаях технически сложных ситуаций выполнения лапароскопических операций. В тоже время данная операция позволит избежать целого ряда серьезных интраоперационных повреждений желчных протоков, позволит снизить частоту раневых инфекций и смертность, уменьшится длительность пребывания пациентов в стационаре. Также при выполнении данной операции сохраняются все принципы миниинвазивной хирургии, что позволяет пациентам после операции быстро реабилитироваться.

7. Cottier D. J. Subtotal cholecystectomy / D. J. Cottier, C. McKay, J. R. Anderson // British Journal of Surgery. - 1991. Vol. 78. - P. 1326-1328.

8. Open versus laparoscopic cholecystectomy: a comparison of postoperative / R. C. Frazee, J. W. Roberts, G. C. Okeson [et al.] // Surgical Endoscopy. - 1991. - Vol. 13. - P. 145-153.

9. Ji W. Role of laparoscopic subtotal cholecystectomy in the treatment of complicated cholecystitis / W. Ji, L.T. Li, J.S. Li // Hepatobiliary \& Pancreatic Diseases International. - 2006. Vol. 5. - P. 584-589.

10. Randomised clinical trial of day-care versus overnight-stay laparoscopic cholecystectomy / M. Johansson, A. Thune, L. Nelvin [et al.] // British Journal of Surgery. - 2006. - Vol. 93. - P. 40-45. 11. Keeling N. J. Laparoscopic exploration of the common bile duct: beyond the learning curve / N. J. Keeling, D. Menzies, R. W. Motson // British Journal of Surgery. - 1999. - Vol. 13. P. 109-112.

12. Lawes D. Anatomical orientation and crosschecking: the key to safer laparoscopic cholecystectomy / D. Lawes, R. W. Motson // British Journal of Surgery. - 2005. - Vol. 92. - P. 663-664.

13. Livingston E. H. A nationwide study of conversion from 
laparoscopic to open cholecystectomy / E. H. Livingston, R. V. Rege // The American Journal of Surgery. - 2004. - Vol. 188. P. 205-211.

14. Laparoscopic cholecystectomy: applicability in the geriatric population / T. H. Magnuson, L. E. Ratner, M. E. Zenilman [et al.] // The American Journal of Surgery. - 1997. - Vol. 6. - P. 9196.

15. Laparoscopic subtotal cholecystectomy in patients with complicated acute cholecystitis or fibrosis / K. Michalowski, P. C. Bornman, J. E. Krige [et al.] // British Journal of Surgery. 1998. - Vol. - 85. - P. 904-906.

16. Perissat J. Laparoscopic cholecystectomy: the European experience / J. Perissat // American Journal of Surgery. - 1993. Vol. 165. - P. 444-449.

17. Ransom K. J. Laparoscopic management of acute cholecystitis with subtotal cholecystectomy / K. J. Ransom //American Journal of Surgery. - 1998. - Vol. 64. - P. 955-957.

18. Rattner D. W. Factors associated with successful laparoscopic cholecystectomy for acute cholecystitis / D. W. Rattner, C. Ferguson, A. L. Warshaw // Annals of Surgery. - 1993. Vol. 217. - P. 233-236.

19. Schafer M. Predictive factors for the type of surgery in acute cholecystitis / M. Schafer, L. Krahenbuhl, M. W. Buchler // American Journal of Surgery. - 2001. - Vol. 182. - P. 291- 297. 20. Schirmer B. D. Laparoscopic cholecystectomy: treatment of choice for symptomatic cholelithiasis / B. D. Schirmer, S. B. Edge, J. Dix // Annals of Surgery. - 1991. - Vol. 213. - P. 665-677.

21. Scott T. R. Laparoscopic cholecystectomy: a review of 12,397 patients / T. R. Scott, K. A. Zucker, R. W. Bailey // Surgical Laparoscopy Endoscopy. - 1992. - Vol. 2. - P. 191-198.

Получено 10.01.12 